\title{
Numerical simulation of a porous-medium type two-dimensional atherosclerosis model
}

\author{
A. Hidalgo ${ }^{1,3, a), b)}$ and L. Tello $\left.2,3, \mathrm{c}\right)$ \\ ${ }^{1}$ Dept. Ingeniería Geológica y Minera. E.T.S.l. Minas y Energía, Universidad Politécnica de Madrid, Rios Rosas 21, \\ 28003 Madrid, Spain. \\ ${ }^{2}$ Dept. Matemática Aplicada. ETS Arquitectura. Universidad Politécnica de Madrid. Av. Juan de Herrera, 28040 \\ Madrid (Spain). \\ ${ }^{3}$ Center for Computational Simulation, Universidad Politécnica de Madrid, Spain.

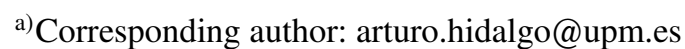 \\ b) arturo.hidalgo@upm.es \\ c)1.tello@upm.es
}

\begin{abstract}
This work is focused on the study of the first stages of atherosclerosis development as an inflammatory disease. The mathematical model on which this research is based is given by a system of two-dimensional nonlinear reaction-diffusion equations with a nonlinear source term in one of the equations, that was proposed originally in [1]. In addition, this model incorporates a nonlinear nonhomogeneous Neumann boundary condition which represents the recruitment of immune cells through the upper boundary as a response to the production of cytokines. In this work a new model is proposed considering nonlinear porous-medium type diffusion. The model is solved using a finite volume scheme with dimension-by-dimension WENO reconstruction in space, using entire polynomials, unlike the pointwise WENO reconstruction commonly used, and a third order Runge-Kutta TVD scheme for time integration. The evolution of the inflammation is studied according to the results of the numerical simulation, depending on the values of the bio-physical parameters and the size of the initial inflammation.
\end{abstract}

\section{INTRODUCTION}

In this work we consider a $2 D$ mathematical model representing the first stages of atherosclerosis disease, which is based on nonlinear reaction-diffusion equations. Atherosclerosis is considered as an inflammatory disease due to the accumulation of cholesterol in the walls of the arteries. This cholesterol is transported by the low density lipoproteins (LDL). A description of the biological process involved can be found, for instance, in [2]. The mathematical model on which this work is based was firstly proposed in [1]. A similar 2D atherosclerosis model was studied in [3]. Motivated by these works, in [4] it was presented and applied a numerical scheme based on an ADER-FV-WENO method to solve the $1 D$ reaction-diffusion model. The numerical simulation was used as well to verify certain properties that were theoretically stated and proved. Recently, in [5], a variant of this mathematical model was introduced incorporating porous-medium type nonlinear diffusion, due the fact that the artery wall is a porous medium, as pointed out in several references which deals with this idea. The aim of this work is to obtain the numerical solution of the $2 D$ mathematical model and describe certain theoretical properties related to the $2 D$ model. The numerical scheme developed in this research is based on the finite volume (FV) method, where a third order Runge-Kutta TVD scheme has been used for time integration and WENO5 for space reconstruction. The two-dimensional WENO reconstruction is performed following the dimension-by-dimension approach, see also $[6,7,8]$, since it is less computationally expensive than the fully $2 D$ WENO reconstruction, obtaining entire polynomials, instead of reconstructing pointwise values, such as in the classical WENO, approach. This way to proceed has the advantage of offering the values and gradients of the polynomials where they are needed, for instance at cell interfaces or Gaussian quadrature points. The drawback of this type of interpolation is that the order of accuracy of the scheme may be reduced, as pointed out in the aforementioned references. 


\section{MATHEMATICAL MODEL}

The $2 D$ mathematical model for the atherosclerosis problem is based on that proposed in [1] reading

$$
\begin{aligned}
& \frac{\partial M}{\partial t}=d_{1} \Delta M^{m}-\lambda_{1} M, \quad(x, y) \in(0, L) \times(0, h), t>0 \\
& \frac{\partial A}{\partial t}=d_{2} \Delta A^{m}+f_{2}(A) M-\lambda_{2} A, \quad(x, y) \in(0, L) \times(0, h), t>0 \\
& \frac{\partial M}{\partial y}(x, 0, t)=\frac{\partial A}{\partial y}(x, 0, t)=0, \quad x \in(0, L), t>0 \\
& \frac{\partial M}{\partial x}(0, y, t)=\frac{\partial A}{\partial x}(0, y, t)=\frac{\partial M}{\partial x}(L, y, t)=\frac{\partial A}{\partial x}(L, y, t)=0, y \in(0, h), t>0 \\
& \frac{\partial M}{\partial y}(x, h, t)=\frac{h}{d_{1}} f_{1}(A) ; \quad \frac{\partial A}{\partial y}(x, h, t)=0, \quad x \in(0, L), t>0 \\
& M(x, y, 0)=M_{0}(x, y), A(x, y, 0)=A_{0}(x, y), \quad(x, y) \in(0,1) \times(0, h) .
\end{aligned}
$$

In (1) $M$ is the concentration of immune cells, $A$ is the concentration of cytokines, $d_{1}$ and $d_{2}$ are diffusion coefficients, $\lambda_{1}$ and $\lambda_{2}$ represent the degradation rates of immune cells and cytokines respectively, $m$ is a positive number. The operator $\Delta$ stands for the Laplacian. There are also the functions $f_{1}(A)$ and $f_{2}(A) M$, both depending on the concentration of cytokines whose expressions are $f_{1}(A)=\frac{\alpha_{1}+\beta_{1} A}{1+\frac{A}{\tau_{1}}}$ and $f_{2}(A)=\frac{\alpha_{2} A}{1+\frac{A}{\tau_{2}}}$, where $\alpha_{1}, \alpha_{2}, \beta_{1}, \beta_{2}, \tau_{1}$ and $\tau_{2}$ are known constants. Since cytokines are secreted by the monocytes and give rise to the recruitment of monocytes, the factor $\beta_{1}$ in the source term of the first equation stands for the auto-amplification factor of the recruitment of monocytes. The term $1+\frac{A}{\tau_{1}}$ represents the effect produced by the fibrous cap, being $\tau_{1}$ the characteristic time of the formation of this fibrous cap, which means the saturation of the recruitment of immune cells. Concerning $1+\frac{A}{\tau_{2}}$ it is the inhibition of the pro-inflammatory cytokines due to the effect of anti-inflammatory cytokines, where $\tau_{2}$ is the characteristic time of this inhibition to take place. All the parameters in the model, $d_{1}, d_{2}, \alpha_{1}, \alpha_{2}, \beta_{1}, \tau_{1}, \tau_{2}, \lambda_{1}$ and $\lambda_{2}$, are assumed positive. It must also be verified that $\tau_{1}>\frac{\alpha_{1}}{\beta_{1}}$. We remark that in the first equation of the system there is no source term, unlike the $1 D$ model, only a degradation one exists, since the recruitment of immune cells takes place through the upper boundary of the domain, where a nonlinear nonhomogeneous boundary condition is given. The domain is the rectangle $[0, L] \times[0, h]$ where $h$ is the width of the artery which is very small compared to the length $L$.

\section{NUMERICAL METHOD}

In this work we apply a numerical scheme to solve the system (1) built in the finite volume framework with third order Runge-Kutta TVD approach for time integration and dimension-by-dimension WENO reconstruction in space, to compute intercell values and gradients. The WENO reconstruction applied here makes use of entire polynomials, as introduced, for instance, in [7,8], instead of the more classical pointwise WENO reconstruction ([9, 10]). This is specially relevant when solving reaction-diffusion problems where gradients of the solution are involved. Previous numerical results for a $1 D$ atherosclerosis model, based on ADER approach, have been reported in $[4,5]$.

We consider the control volume $I_{i j}=\left[x_{i-\frac{1}{2}}, x_{i+\frac{1}{2}}\right] \times\left[y_{j-\frac{1}{2}}, y_{j+\frac{1}{2}}\right]$ and integrate the system (1) dividing by $\Delta x_{i} \times \Delta y_{j}$ where $\Delta x_{i}=x_{i+\frac{1}{2}}-x_{i-\frac{1}{2}}$ and $\Delta y_{j}=y_{j+\frac{1}{2}}-y_{j-\frac{1}{2}}$ to get

$$
\frac{d \mathbf{u}_{i, j}(t)}{d t}=\frac{1}{\Delta x_{i}} \int_{y_{j-\frac{1}{2}}}^{y_{j+\frac{1}{2}}}\left(\mathbf{F}_{i+\frac{1}{2}, j}-\mathbf{F}_{i-\frac{1}{2}, j}\right) d y+\frac{1}{\Delta y_{j}} \int_{x_{i-\frac{1}{2}}}^{x_{i+\frac{1}{2}}}\left(\mathbf{G}_{i, j+\frac{1}{2}}-\mathbf{G}_{i, j-\frac{1}{2}}\right) d x+\overline{\mathbf{R}}_{i, j}
$$

where $\mathbf{u}=(M, A)^{T}$ is the vector of unkowns, $\mathbf{F}=-\left(d_{1} \frac{\partial M^{m}}{\partial x}, d_{2} \frac{\partial A^{m}}{\partial x}\right)^{T}, \mathbf{G}=-\left(d_{1} \frac{\partial M^{m}}{\partial y}, d_{2} \frac{\partial A^{m}}{\partial y}\right)^{T}$ is the vector of physical fluxes in $x$ and $y$ directions respectively and $\overline{\mathbf{R}}_{i, j}=\frac{1}{\Delta x_{i} \Delta y_{j}} \int_{x_{i-\frac{1}{2}}}^{x_{i+\frac{1}{2}}} \int_{y_{j-\frac{1}{2}}}^{y_{j+\frac{1}{2}}} \mathbf{R}(x, y, t) d y d x$ is the cell average of the source term. 
In order to obtain the fluxes appearing in the expression (2), we apply a dimension-by-dimension WENO reconstruction procedure. This technique consists of performing two $1 D$ WENO reconstructions, for both Cartesian directions. Usually it is done in $x$ direction and afterwards it is performed in $y$ direction applied to each single coefficient of the polynomials in $x$ direction. For details on this type of reconstruction we refer the reader to [7, 8, 6] where a detailed description is given. Concerning time integration we use a third order Runge-Kutta TVD, see [11].

\section{NUMERICAL RESULTS}

In the following example we apply the numerical scheme (2) to solve the problem (1) using the values of the parameters given in table 1 for different values of the exponent $m$, namely $m=1,2,3$.

TABLE 1. Parameters for the numerical simulation

\begin{tabular}{cccccccccc}
\hline$L=1$ & $\alpha_{1}$ & $\alpha_{2}$ & $\beta_{1}$ & $\tau_{1}$ & $\tau_{2}$ & $\lambda_{1}$ & $\lambda_{2}$ & $d_{1}$ & $d_{2}$ \\
\hline & 1 & 8 & 1 & $42 / 43$ & 1 & 1 & $10^{-5}$ & $10^{-4}$ \\
\hline
\end{tabular}

In all cases we use the same initial data given by the expression

$$
\begin{aligned}
& M(x, y, 0)= \begin{cases}-700 e^{x^{2}}(x-0.4)(x-0.6)+2 & \text { if } x \in[0.4,0.6] \\
2 & \text { if } x \notin[0.4,0.6]\end{cases} \\
& A(x, y, 0)=\left\{\begin{array}{ll}
-600 e^{x^{2}}(x-0.4)(x-0.6)+2 & \text { if } x \in[0.4,0.6] \\
0 & \text { if } x \notin[0.4,0.6]
\end{array} \quad \forall(x, y) \in[0,1] \times\left[0,10^{-3}\right] .\right.
\end{aligned}
$$

Results of the simulation are depicted in Figure 1 and 2 (left plot), where the distribution of immune cells for different values of the exponent $m$ are applied $(m=1,2,3)$. The FV mesh used is formed by $50 \times 20$ regular control volumes. The results show that, in the nonlinear cases $(m=2$ and $m=3)$ the propagation front becomes sharper than in the linear case $(m=1)$, where a smoother solution is attained. This happens usually in porous-media problems as put forward, for instance in [12], where ADER approach for nonlinear reaction-diffusion problems is applied.
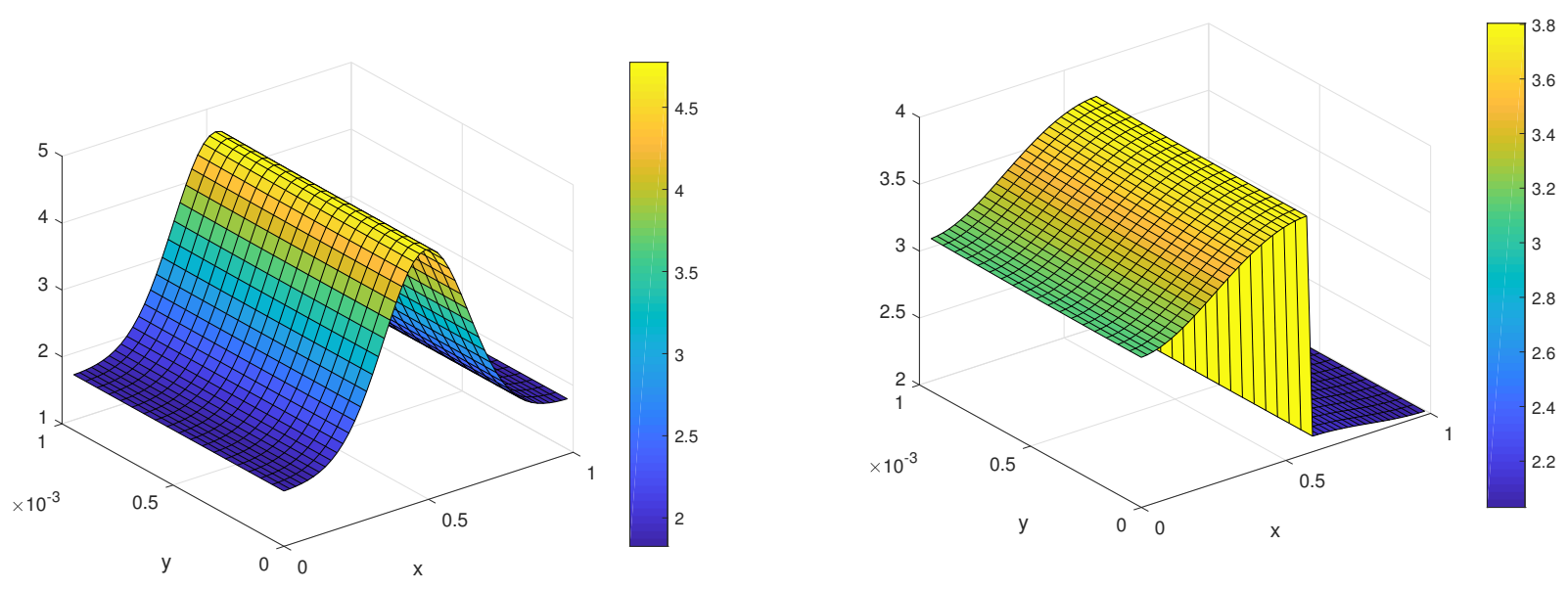

FIGURE 1. Spatial distribution of immune cells $(M)$ for $t=0.1$. Left: linear case (that is $m=1$ ), right nonlinear case when $m=2$

In order to validate the numerical scheme developed, we have solved an auxiliary problem with known analytical solution $M(x, y, t)=80 t x^{2}(x-1)^{2} y^{2} \exp \left(-3 x^{2}-3 y^{2}\right)$ and $A(x, y, t)=100(1+t) x^{2}(x-1)^{2} y^{2}\left(y-10^{-3}\right)^{2} \exp \left(-x^{2}-y^{2}\right)$. The result is shown in figure 2 (right plot) where, in order to visualize the comparison between the exact and numerical solution, a $2 D$ cut of the $3 D$ plot for $x=0.5$ is shown. The $L_{2}$ norm of the error achieved is $\|\epsilon\|_{L_{2}}=1.46 \times 10^{-6}$. 

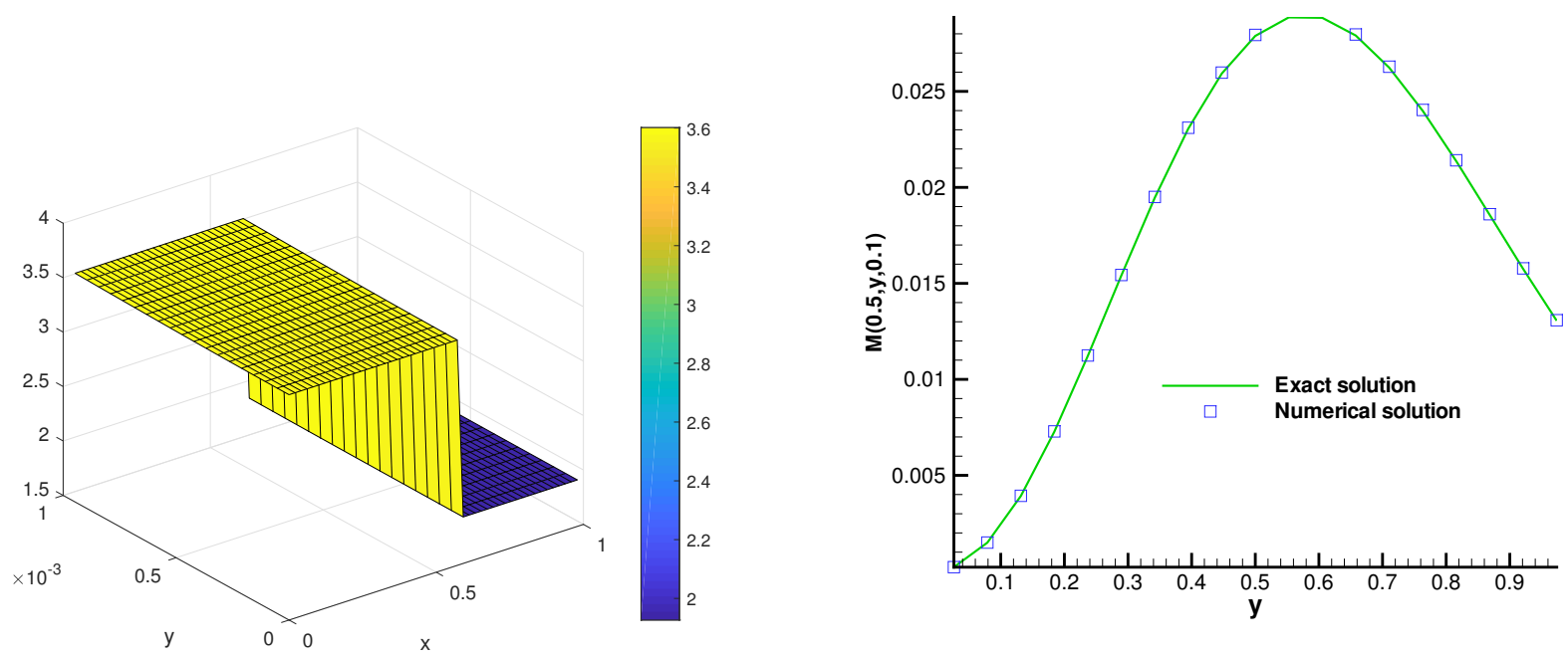

FIGURE 2. Left: Spatial distribution of immune cells $(M)$ for $t=0.1$ when $m=3$. Right: Comparison of exact solution of the auxiliary problem with the numerical solution taking $m=3$ cutting the 3D plot with the plane $x=0.5$ for the output time $t=0.1$

\section{Conclusions}

In this work we have considered a $2 D$ reaction-diffusion model representing the first stages of atherosclerosis disease. In the model nonlinear porous-medium type diffusion is considered and it is incorporated a nonlinear nonhomogeneous boundary condition, representing the recruitment of monocytes through the boundary. With the aim of obtaining a numerical solution of the proposed problem, a finite volume scheme with dimension-by-dimension WENO reconstruction and third order Runge-Kutta TVD scheme for time integration. Validation of the model is assessed by means of a manufactured solution.

\section{ACKNOWLEDGMENTS}

This work is partially supported by the research projects MTM2017-85449-P and MTM2017-83391-P of Ministerio de Economía, Industria y Competitividad (Spain).

\section{REFERENCES}

[1] N. El Khatib, S. Genieys and V. Volpert, Math. Model. Nat. Phenom. 2, 126-141 (2007).

[2] P. Libby, Nature 420, 19-26 (2002).

[3] N. El Khatib, S. Genieys, B. Kazmierczak and V. Volpert, Journal of Mathematical Biology 65, 349-374 (2012).

[4] A. Hidalgo, L. Tello and E.F. Toro, Journal of Mathematical Biology 68, 1785-1814 (2014).

[5] A. Hidalgo and L. Tello, Computers\&Fluids 169, $380-387$ (2018).

[6] V. Titarev and E. Toro, Journal of Computational Physics 201, 238 - 260 (2004).

[7] M. Dumbser, O. Zanotti, A. Hidalgo, and D. S. Balsara, Journal of Computational Physics 248, 257 - 286 (2013).

[8] M. Dumbser, A. Hidalgo, and O. Zanotti, Computer Methods in Applied Mechanics and Engineering 268, $359-387$ (2014).

[9] X.-D. Liu, S. Osher, and T. Chan, Journal of Computational Physics 115, 200 - 212 (1994).

[10] G.-S. Jiang and C.-W. Shu, Journal of Computational Physics 126, $202-228$ (1996).

[11] S. Gottlieb and C.-W. Shu, Mathematics of Computation 67, 73-85 (1998).

[12] E. F. Toro and A. Hidalgo, Applied Numerical Mathematics 59, 73 - 100 (2009). 